\title{
Hallazgos imagenológicos de la hemosiderosis pulmonar idiopática en el adulto: una revisión de la literatura de los últimos 20 años
}

\author{
Julián Andrés Muñoz Durán* \\ Ana Fernanda Muñoz Durán**
}

\begin{abstract}
*Residente de Radiología. Médico cirujano. Universidad Industrial de Santander. Bucaramanga. Santander. Colombia
**Estudiante de Medicina. Fundación Universitaria San Martín. Sabaneta. Antioquia. Colombia

Correspondencia: Dr. Julián Andrés Muñoz Durán. Carrera 76 No. 53-79 Torre 4 apartamento 1113. Conjunto residencial Paraíso de colores. Medellín. Antioquia. Colombia. Tel:(+57) 301371 7275. E-mail: jumudu@gmail.com
\end{abstract}

Resumen

La hemosiderosis pulmonar idiopática es una entidad rara caracterizada por hemorragia alveolar capilar. Su tríada clásica es hemoptisis crónica o recurrente, anemia por deficiencia de hierro y opacidades en las imágenes pulmonares. El objetivo de esta revisión fue determinar la frecuencia en radiografía y tomografía de tórax, de los hallazgos de hemosiderosis pulmonar idiopática en adultos, reportados en la literatura durante los últimos 20 años, de acuerdo con los hallazgos semiológicos en imagen, localización y distribución. Se hizo una búsqueda de publicaciones en bases de datos. Se seleccionaron 42 estudios, se estratificaron variables y se recopilaron los hallazgos. La consolidación y las opacidades reticulonodulares fueron el hallazgo más frecuente en radiografía. En tomografía el hallazgo principal fue el vidrio deslustrado en la región basal. No hubo diferencias en los hallazgos semiológicos de imagen en cuanto al compromiso de acuerdo con el género, aunque las mujeres tendieron a presentar una distribución difusa. MÉD.UIS.2020;33(2):55-64.

Palabras clave: Hemosiderosis. Radiografía torácica. Anemia. Disnea. Tomografía.

\section{Imaging findings of idiopathic pulmonary hemosiderosis in adults: Literature review of the last 20 years}

\begin{abstract}
Idiopathic pulmonary hemosiderosis is a rare entity characterized by capillary alveolar hemorrhage. Its classic triad is chronic or recurrent hemoptysis, iron deficiency anemia, and opacities in lung images. The objective of this review was to determine the frequency in chest radiography and chest tomography of the findings of idiopathic pulmonary hemosiderosis in adults, reported in the literature during the last 20 years, according to the semiological imaging findings, location and distribution. A search of publications in databases was made. Forty-two studies were collected, variables were stratified in different categories and the findings were compiled. The most frequent finding in chest radiography were consolidation and reticulonodular opacities. On the other hand, the main finding in tomography was ground glass opacities in the basal region. There were no differences in the pattern of compromise by gender, although women tended to have a more diffuse distribution. MÉD.UIS.2020;33(2):55-64.
\end{abstract}

Keywords: Hemosiderosis. Chest radiography. Anemia. Dyspnea. Tomography.

¿Cómo citar este artículo?: Muñoz Durán JA, Muñoz Durán AF. Hallazgos imagenológicos de la hemosiderosis pulmonar idiopática en el adulto: una revisión de la literatura de los últimos 20 años. MÉD.UIS.2020;33(2):55-64. doi: 10.18273/revmed.v33n2-2020010 


\section{Introducción}

La hemosiderosis pulmonar idiopática es una enfermedad rara con una incidencia reportada de 0,24-1,26 casos por millón de habitantes en la literatura científica sueca y japonesa respectivamente ${ }^{1,2,3}$. Hoy en día, no existen publicaciones acerca de la incidencia y prevalencia en Colombia. Descrita inicialmente por Virchow en 1864 como induración marrón esencial del pulmón ${ }^{3}$, posteriormente caracterizada por Ceelen en 1921, quien publicó los resultados de una autopsia realizada a dos niños con grandes cantidades de hemosiderina en los pulmones ${ }^{3,4}$ y luego diagnosticada ante mortem por Waldenstrom en $1944^{3}$; es una entidad cuya precisión etiológica permanece desconocida, caracterizada por hemorragia alveolar capilar, resultando en el depósito y acumulación de hemosiderina en los pulmones $^{2}$. Su triada clásica es la de hemoptisis crónica o recurrente, anemia por deficiencia de hierro y hallazgos imagenológicos, todos ellos secundarios a hemorragia pulmonar ${ }^{2,5}$. Usualmente diagnosticada en la infancia ( $80 \%$ de los casos) y comúnmente antes de los 10 años, con curso severo y pobre pronóstico en este grupo etario; en los adultos (20\% de los casos) se diagnostica antes de los 30 años, con una ligera predilección por el género masculino, presentando un curso crónico con agudizaciones, síntomas más leves y un pronóstico favorable, sin embargo esta condición puede progresar a fibrosis pulmonar con falla respiratoria y cor pulmonale ${ }^{2}$.

Aunque la fisiopatología permanece sin conocerse, se han propuesto algunas hipótesis de su origen, como la teoría autoinmune, por su frecuente asociación con enfermedad celíaca (llamada síndrome de Lane Hamilton), dermatitis herpetiforme, artritis reumatoide o glomerulonefritis. Otra probable explicación se fundamenta en la etiología ambiental, que da explicación a partir de la exposición a toxinas del hongo Stachybotrys chartarum que tiene efectos sobre la vasculatura alveolar ${ }^{6}$. Según la teoría alérgica, se sustenta en la asociación con la hipersensibilidad a la leche de vaca y finalmente la teoría genética tiene su justificación en la predisposición hereditaria a sufrir la enfermedad. ${ }^{2}$

Las características de imagen de la hemosiderosis pulmonar idiopática no son específicas y no hay manifestaciones radiológicas patognomónicas. Las estructuras afectadas primariamente corresponden al parénquima pulmonar, sin embargo, no se debe desconocer que la pleura también podría tener afección secundaria. Aunque los rayos $X$ de tórax son la prueba inicial de diagnóstico, la tomografía computarizada de alta resolución es más sensible y puede demostrar anormalidades no visibles en radiografía convencional de tórax. Manifestaciones como adenopatías y derrame pleural son infrecuentes ${ }^{2}$.

Dado el anterior contexto, se hace necesario identificar los hallazgos más frecuentes en la población adulta de la hemosiderosis pulmonar idiopática. Por lo tanto, el objetivo de este artículo es determinar la frecuencia en radiografía y tomografía computarizada de tórax de los hallazgos de hemosiderosis pulmonar idiopática de acuerdo con las características de imagen, localización y distribución, así como establecer diferencias según el género.

\section{Metodología de búsqueda}

\section{Estrategia de búsqueda y criterios de revisión}

En junio del 2019 se realiza una búsqueda con el término "Idiopathic Pulmonary Hemosiderosis" en MEDLINE, PubMed, LILACS, SciELO y OVID, limitando la misma al periodo comprendido entre enero de 1999 y diciembre de 2019, incluyendo los reportes de caso de la población adulta, sin exclusión del idioma, que indicaran hallazgos radiológicos o tomográficos de hemosiderosis pulmonar idiopática. Se excluyen los casos de pacientes pediátricos, sin embargo, en algunos artículos se expone más de un caso, y cuando alguno de ellos hace referencia a población pediátrica se prescinde de este y únicamente se tienen en cuenta para la presente revisión los casos en adultos. Para detalles de la búsqueda, (Ver flujograma 1). Se eliminaron los casos de pacientes pediátricos, dado que la literatura acerca de este tema, en este grupo etario es amplia y la evolución clínica es diferente a los adultos.

Una vez filtrados, se encontraron un total de 46 artículos en 9 idiomas diferentes: inglés, español, chino, japonés, alemán, portugués, francés, ruso y rumano, los cuales fueron traducidos mediante software de traducción de Google. Algunos fueron presentados como revisión de la literatura, cartas al editor, y otros como estudio original en la correspondiente publicación, sin embargo, fueron clasificados por las bases de datos como reportes de caso (Ver Tabla 1). Hubo 4 artículos los cuales no fue posible encontrarlos para su uso. Finalmente se 
realiza la revisión con 42 publicaciones (Ver Tabla 2), siendo la mayoría en inglés y correspondientes al año 2008 (7 publicaciones $-16,6 \%$ ).

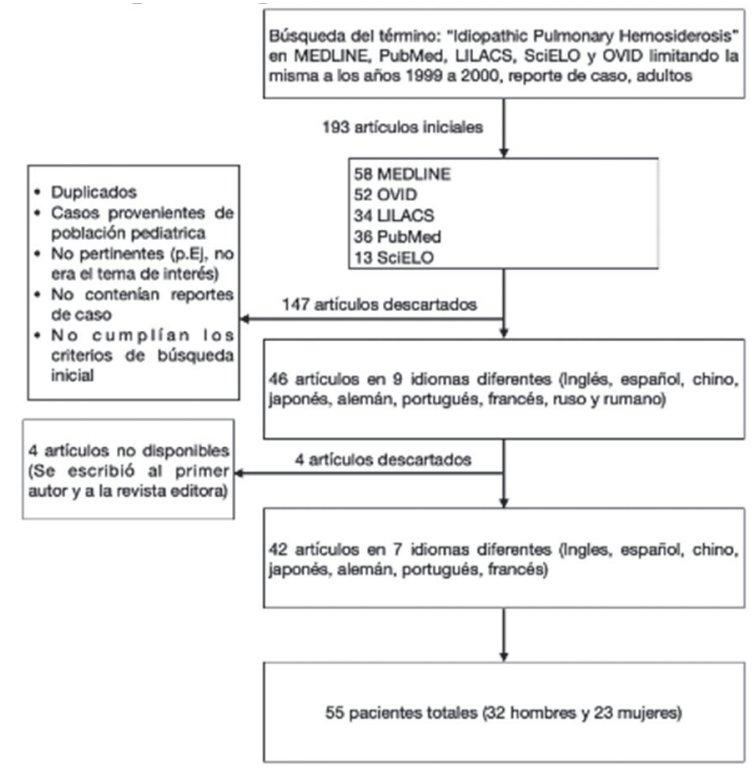

Flujograma 1. Estrategia de búsqueda

Fuente: autores.

Tabla 1. Tipos de publicación usados en el análisis

\begin{tabular}{|l|l|l|}
\hline Tipo de articulo & $\begin{array}{l}\text { Número de artículos } \\
\text { encontrados }\end{array}$ & Porcentaje (\%) \\
\hline Reporte de caso & 37 & 88,1 \\
\hline Carta al editor & 3 & 7,1 \\
\hline Investigación Original & 2 & 4,8 \\
\hline
\end{tabular}

Fuente: autores.

De estos, se extrajeron, el año, idioma y tipo de publicación, método final de diagnóstico de hemosiderosis pulmonar idiopática, edad del paciente, hallazgos de imagen que el autor exponía de manera textual tanto en el cuerpo del artículo como en el pie de foto que acompañaba el hallazgo radiográfico y/o tomográfico en caso de haberlos. Se excluyeron los hallazgos descritos en los pacientes cuando estaban en la infancia, sin excluir las descripciones de la edad adulta, como el artículo de loachimanescu et al $^{3}$ en el cual se hace un seguimiento en el tiempo. En Vinodh et al..$^{31}$, se analiza uno de los dos casos expuestos debido a que un paciente es pediátrico y no cumple con los criterios de la presente revisión. No se tuvo en cuenta los hallazgos en resonancia magnética, a pesar de ser evaluadas para la extracción de datos, esto último debido a que la técnica de imagen para la evaluación del parénquima pulmonar es más limitada que en la radiografía o en la tomografía. Patrucco et al ${ }^{13}$, usaron la resonancia magnética para la identificación de focos de miocarditis en pacientes con hemosiderosis pulmonar idiopática.

\section{Análisis estadístico}

El análisis estadístico fue realizado utilizando Excel software (Versión 16.17). Se realiza conteo del número de estudios, idiomas de las publicaciones, forma de establecer el diagnóstico y adicionalmente se cuantifican las frecuencias de los hallazgos, la distribución y la localización de estos. Posteriormente se realiza análisis para establecer cuáles son los hallazgos más frecuentes en radiografía y tomografía de la hemosiderosis pulmonar idiopática en adultos. Adicionalmente se establece la edad promedio de la población, rango y mediana de edad y porcentaje de género.

La estratificación de los hallazgos radiológicos se hizo a través de subcategorías, tradicionalmente descritas, vale la pena mencionar que se empleó el uso de terminología de la sociedad Fleishner 45 y las descripciones de libros clásicos de literatura radiológica. Para los hallazgos de imagen radiográfica se asumieron las categorías descritas por el doctor Richard Webb ${ }^{46}$ :

\section{Hallazgos semiológicos}

Que determina la plantilla básica de la anormalidad producido por la enfermedad y su compromiso de acuerdo con el sitio anatómico (Ver Figura 1 y 2):

- Consolidación: Describe la ocupación del espacio alveolar o reemplazo de este.

- Lineal o septal: Resulta de la presencia de líneas A y $B$ de Kerley por engrosamiento de septos interlobulillares.

- Reticular: Múltiples líneas que se intersecan a menudo de apariencia irregular, delineadas por lo que aparentan ser espacios irregulares o redondos, en el que no predomina el engrosamiento septal.

- Nodular: Presencia de algunos nódulos en el parénquima pulmonar.

- Reticulonodular: Patrón reticular con nódulos

- Opacidades en vidrio deslustrado: Aumento de la opacidad del pulmón sin franca consolidación, con lo que se pueden ver las estructuras vasculares pulmonares.

- Crazy Paving, adoquín o pavimento: Sumatoria de vidrio deslustrado y opacidades intersticiales (inter e intralobulillares). 
Tabla 2. Publicaciones incluidas en el análisis

\begin{tabular}{|c|c|c|c|c|}
\hline Estudio & Año Publicación & Tipo de publicación & Idioma de Publicación & Autor \\
\hline 1 & 2018 & Reporte de caso & Inglés & Yanagihara $\mathrm{T} \mathrm{et}^{\mathrm{P}}{ }^{7}$ \\
\hline 2 & 2017 & Reporte de caso & Español & Patiño et al ${ }^{8}$ \\
\hline 3 & 2017 & Carta al editor & Inglés & Silva P, Ferreira PG ${ }^{9}$ \\
\hline 4 & 2016 & Reporte de caso & Inglés & Popp A et al. ${ }^{10}$ \\
\hline 5 & 2016 & Reporte de caso & Inglés & Berger et al ${ }^{6}$ \\
\hline 6 & 2016 & Reporte de caso & Español & Barrera - Vargas ${ }^{11}$ \\
\hline 7 & 2013 & Reporte de caso & Inglés & Cambruzzi et al ${ }^{12}$ \\
\hline 8 & 2013 & Reporte de caso & Inglés & Patrucco F et al ${ }^{13}$ \\
\hline 9 & 2012 & Reporte de caso & Inglés & Töro et al ${ }^{14}$ \\
\hline 10 & 2012 & Carta al editor & Portugués & Santos et al ${ }^{15}$ \\
\hline 11 & 2011 & Estudio retrospectivo & Inglés & Miwa et al ${ }^{16}$ \\
\hline 12 & 2011 & Reporte de caso & Chino & Lang et al ${ }^{17}$ \\
\hline 13 & 2011 & Estudio Prospectivo & Inglés & Persson HL et al ${ }^{18}$ \\
\hline 14 & 2010 & Reporte de caso & Español & Oviedo et al ${ }^{19}$ \\
\hline 15 & 2010 & Reporte de caso & Alemán & Krumsiek A et al20 \\
\hline 16 & 2009 & Carta al editor & Español & Robles et al ${ }^{21}$ \\
\hline 17 & 2008 & Reporte de caso & Chino & Mu XD et al ${ }^{22}$ \\
\hline 18 & 2008 & Reporte de caso & Inglés & Harte et $a^{23}$ \\
\hline 19 & 2008 & Reporte de caso & Inglés & Foglia LM - Deering S ${ }^{24}$ \\
\hline 20 & 2008 & Reporte de caso & Portugués & Souza et al ${ }^{25}$ \\
\hline 21 & 2008 & Reporte de caso & Inglés & Mayes et al ${ }^{26}$ \\
\hline 22 & 2008 & Reporte de caso & Inglés & Allen et a ${ }^{27}$ \\
\hline 23 & 2008 & Reporte de caso & Inglés & Chen et al ${ }^{28}$ \\
\hline 24 & 2007 & Reporte de caso & Japonés & Inayama et al ${ }^{29}$ \\
\hline 25 & 2007 & Reporte de caso & Inglés & Agarwal $R$ et al ${ }^{1}$ \\
\hline 26 & 2007 & Reporte de caso & Inglés & Deniz $\mathrm{O}$ et $\mathrm{al}^{30}$ \\
\hline 27 & 2007 & Reporte de caso & Inglés & Gencer $\mathrm{M}$ et al ${ }^{5}$ \\
\hline 28 & 2006 & Reporte de caso & Inglés & Vinodh et al ${ }^{31}$ \\
\hline 29 & 2006 & Reporte de caso & Inglés & loachimanescu-Jennings ${ }^{32}$ \\
\hline 30 & 2005 & Reporte de caso & Inglés & Malhotra ${ }^{33}$ \\
\hline 31 & 2005 & Reporte de caso & Inglés & Soto - Soares ${ }^{34}$ \\
\hline 32 & 2005 & Reporte de caso & Francés & Maalej et al ${ }^{35}$ \\
\hline 33 & 2004 & Reporte de caso & Inglés & loachimescu et al ${ }^{3}$ \\
\hline 34 & 2004 & Reporte de caso & Inglés & Turay et al ${ }^{36}$ \\
\hline 35 & 2003 & Reporte de caso & Inglés & Helman et $\mathrm{al}^{37}$ \\
\hline 36 & 2002 & Reporte de caso & Inglés & Calabrese et a ${ }^{38}$ \\
\hline 37 & 2001 & Reporte de caso & Inglés & Tedeschi et al ${ }^{39}$ \\
\hline 38 & 2000 & Reporte de caso & Japonés & Kishimoto - Kondou ${ }^{40}$ \\
\hline 39 & 2000 & Reporte de caso & Inglés & Ruchin et al ${ }^{14}$ \\
\hline 40 & 2000 & Reporte de caso & Inglés & Godoy et al ${ }^{42}$ \\
\hline 41 & 2000 & Reporte de caso & Inglés & Bravry et al ${ }^{43}$ \\
\hline 42 & 1999 & Reporte de caso & Español & Ramirez et al ${ }^{44}$ \\
\hline
\end{tabular}

Fuente: autores. 
Mayo - agosto
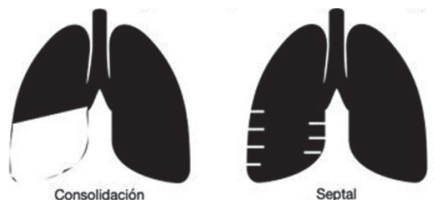

Septal
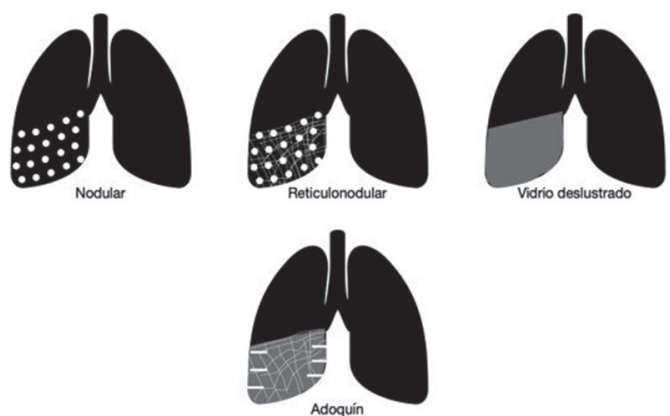

Figura 1. Hallazgos semiológicos de imagen en radiografía

Fuente: autores.
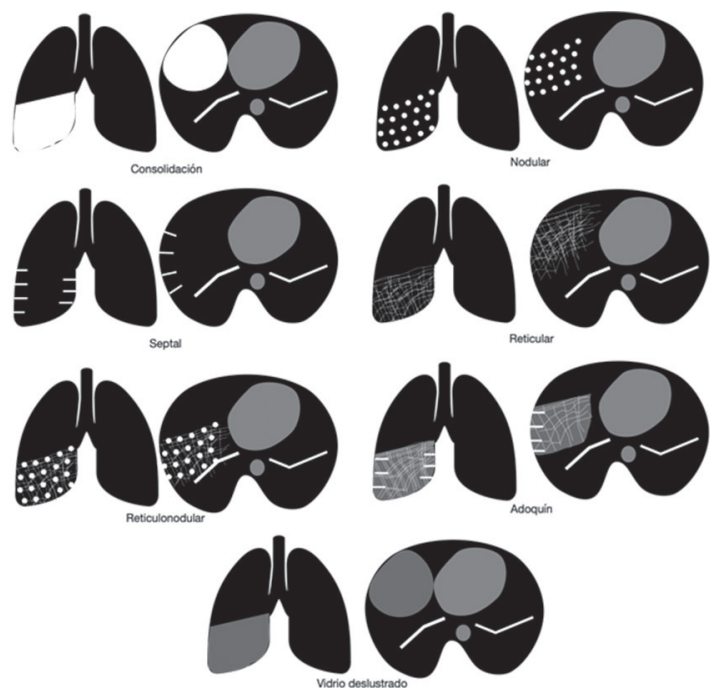

Figura 2. Hallazgos semiológicos de imagen en tomografía.

Fuente: autores.

Dado que en algunas descripciones no existía especificidad de los hallazgos de acuerdo con las categorías mencionadas anteriormente $\mathrm{o}$ simplemente eran evocadas como "infiltrados" u "opacidades" sin determinar el sitio anatómico, se hizo necesaria la interpretación de las imágenes disponibles y la actualización con la terminología por parte de los autores, reflejando la evolución natural de la literatura médica.

\section{Distribución}

De acuerdo a la disposición del hallazgo dominante. Algunas descripciones no hacían referencia a los lóbulos pulmonares en específico, se analizaron las radiografías dividiendo los campos pulmonares en tercios de acuerdo con la extensión (superior, medio e inferior). Como en la mayoría de las ocasiones no se contaba con la proyección lateral y no siempre los hallazgos eran secundarios a consolidación, es difícil determinar exactamente la localización anatómica. En radiografía, en caso de haber compromiso de todo el pulmón se clasifica en difuso (Ver Figura 3), y en tomografía, el compromiso perihiliar con respeto periférico se catalogó como central.
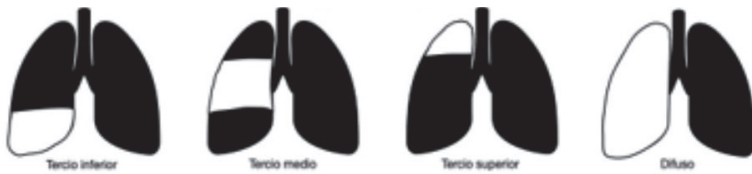

Figura 3. Distribución de los hallazgos en radiografía.

Fuente: autores.

\section{Localización}

Hace referencia a la ubicación del compromiso pulmonar de acuerdo con la lateralidad, derecha, izquierda, unilateral o bilateral.

\section{Otros hallazgos}

Estos incluyen, estudios normales, adenopatías, panalización o presencia de quistes, otros hallazgos extrapulmonares que fueron descritos por los autores se tabularon, pero dado que no tenían representación ni relación con la enfermedad, tales como neumotórax o derrame pleural, no se tuvieron en cuenta.

\section{Desarrollo del tema}

De los 42 estudios, $37(88,1 \%)$ eran reportes de casos, seguidos de 3 cartas al editor $(7,1 \%), 1$ estudio retrospectivo $(2,4 \%)$ y otro prospectivo $(2,4 \%)$. El idioma predominante fue el inglés con 69\% (29/42) seguido por español con 11,9\%, (5/42), menos frecuentemente chino, japonés y portugués, cada una representando el 4,8\% ( 2 estudios por cada idioma), finalmente, francés y alemán con 1 estudio respectivamente constituyendo el $2,4 \%$ cada uno. La mayoría de las publicaciones, databan del año 2008 con 7 artículos, representando el 16,7\%. (Ver Tabla 2).

\section{Características de la población}

Se obtuvo un total de 55 pacientes: 32 hombres $(58,2 \%)$ y 23 mujeres ( $41,8 \%)$; la edad promedio fue de 36,2 años, con un rango que oscilaba entre los 18 
y 76 años, de acuerdo con los criterios de búsqueda establecidos. La mediana de la edad fue 32 años. La forma de realizar el diagnóstico definitivo fue mediante biopsia en 40 pacientes $(72,7 \%)$, lavado broncoalveolar en 10 pacientes $(18,2 \%$ ) y necropsia en 2 pacientes (3,6\%). Otras formas descritas en los artículos son examen de esputo, síntomas clínicos, laboratorio al descartar otras causas y un artículo que lo presenta como "extensamente estudiado en una institución" por lo cual se desconoce la forma exacta del diagnóstico, todas estas con 1,8\% (Ver Tabla 3).

Tabla 3. Forma de diagnóstico definitivo

\begin{tabular}{|l|l|l|}
\hline Diagnóstico definitivo & $\begin{array}{l}\text { No de } \\
\text { pacientes }\end{array}$ & $\begin{array}{l}\text { Porcentaje } \\
\text { (\%) }\end{array}$ \\
\hline Biopsia & 40 & 72,7 \\
\hline Lavado broncoalveolar & 10 & 18,2 \\
\hline Examen de esputo & 1 & 1,8 \\
\hline Desconocida & 1 & 1,8 \\
\hline $\begin{array}{l}\text { Clínica y Laboratorio (Descartaron } \\
\text { otras causas) }\end{array}$ & 1 & 1,8 \\
\hline Autopsia & 2 & 3,6 \\
\hline
\end{tabular}

Fuente: autores.

\section{Hallazgos en radiografía de tórax}

Se reportaron un total de 39 radiografías (19 en hombres y 20 en mujeres). El hallazgo semiológico de imagen más frecuente en los estudios de radiografía de tórax fue la consolidación (35,9\%), seguido de opacidades reticulonodulares (20,5\%), opacidades reticulares y opacidades en vidrio deslustrado (Ver Tabla 4). Se encontraron otros hallazgos que, si bien fueron descritos, salen del interés primario de este artículo, ya que son descripciones por fuera del parénquima pulmonar. Hay que destacar que en muchas ocasiones se mencionan varios hallazgos semiológicos en un mismo estudio, por ejemplo, consolidación y áreas de vidrio deslustrado.

Tabla 4. Frecuencia de los hallazgos radiográficos.

\begin{tabular}{|l|c|}
\hline Hallazgo semiológico & Porcentaje (\%) \\
\hline Consolidación & 35,9 \\
\hline Reticulonodular & 20,5 \\
\hline Reticular & 12,8 \\
\hline Vidrio deslustrado & 10,3 \\
\hline $\begin{array}{l}\text { Otros hallazgos (p. Ej: engrosamiento } \\
\text { pleural, neumotórax, adenopatía, } \\
\text { calcificación, congestión hiliar, } \\
\text { cardiomegalia) o simplemente } \\
\text { normales. }\end{array}$ & 20,5 \\
\hline
\end{tabular}

Fuente: autores.
Se hizo un análisis adicional separando los hallazgos de hombres y mujeres, lo que permitió encontrar a la consolidación como el hallazgo predominante en ambos géneros (Ver Figura 4).

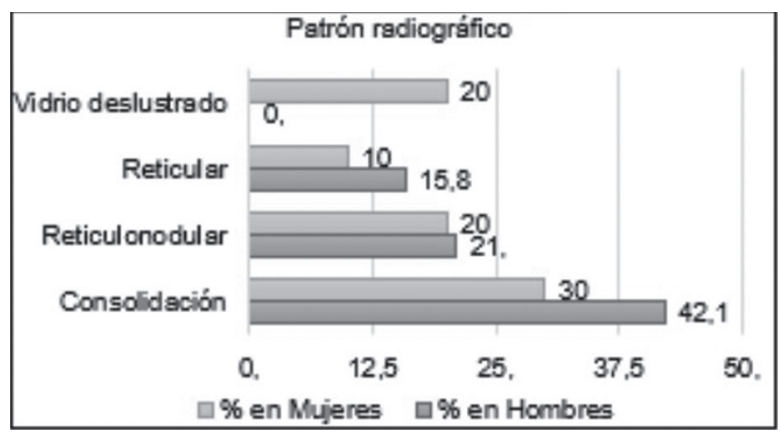

Figura 4. Cuantificación de los hallazgos de acuerdo al género.

Fuente: autores.

En cuanto a la localización y la distribución de las anormalidades en la radiografía de tórax, se halló que las localizaciones más frecuentes estuvieron en el tercio inferior $(46,2 \%)$ y de manera difusa $(30,8 \%)$ (Ver Tabla 5).

Tabla 5. Distribución de las anormalidades en la radiografía

\begin{tabular}{|l|c|}
\hline Distribución & Porcentaje (\%) \\
\hline Difuso & 30,8 \\
\hline Superior & 7,7 \\
\hline Medio & 7,7 \\
\hline Inferior & 46,2 \\
\hline
\end{tabular}

Fuente. autores.

Encontrando una distribución difusa y en el tercio inferior con más frecuencia en las mujeres. En los hombres el predominio fue la localización inferior (Ver Figura 5).

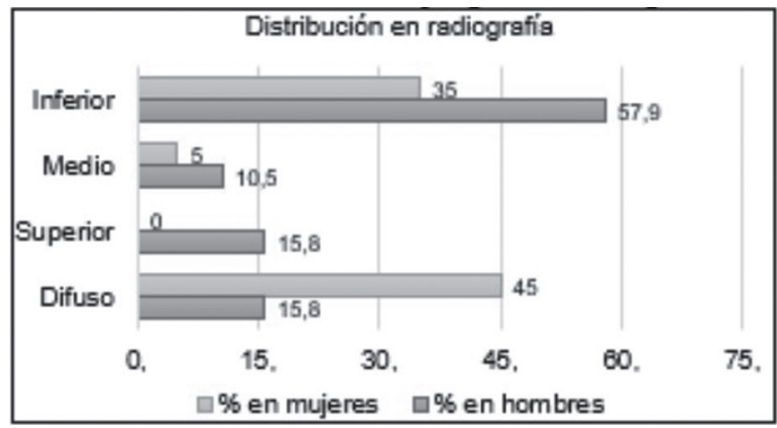

Figura 5. Distribución de las anormalidades por género en radiografía.

Fuente: autores. 
Al elaborar un análisis de acuerdo con la localización, lo más frecuente fue el compromiso bilateral, con más de la mitad de los casos; hallazgos similares se encontraron en hombres y mujeres (Ver Figura 6).

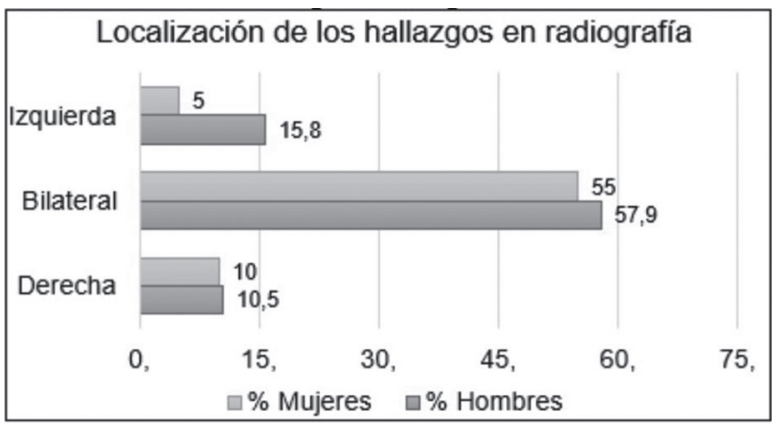

Figura 6. Localización de los hallazgos en radiografía.

Fuente: autores.

En otros hallazgos, se encontró que hasta el $5 \%$ de las radiografías eran catalogadas como normales y solo se reportó un engrosamiento pleural en el artículo de Godoy et al.42, lo cual también se encuentra descrito en la literatura ${ }^{47}$.

\section{Hallazgos en tomografía}

Se reportaron 45 tomografías (27 hombres y 18 mujeres). Se realizó el mismo procedimiento para el análisis de los estudios radiográficos, se encontró entonces que el hallazgo más frecuente por tomografía de la hemosiderosis pulmonar idiopática, es la presencia de opacidades en vidrio deslustrado presente en $64,4 \%$ de los casos, seguida de la consolidación en el $40 \%$ de los casos. Consideraciones similares se encontraron al hacer el análisis por género (Ver Tabla 6).

Tabla 6. Hallazgos por tomografía.

\begin{tabular}{|l|l|l|l|}
\hline $\begin{array}{l}\text { Hallazgo } \\
\text { semiológico en } \\
\text { tomografía }\end{array}$ & $\begin{array}{l}\text { Porcentaje } \\
\text { general (\%) }\end{array}$ & $\begin{array}{l}\text { Porcentaje en } \\
\text { hombres (\%) }\end{array}$ & $\begin{array}{l}\text { Porcentaje } \\
\text { en mujeres } \\
\text { (\%) }\end{array}$ \\
\hline Vidrio deslustrado & 64,4 & 74,1 & 50 \\
\hline Consolidación & 40 & 55,5 & 16,7 \\
\hline $\begin{array}{l}\text { Micronodular } \\
\text { (Tamaño entre 2-7 } \\
\text { mm) }\end{array}$ & 6,7 & 11,1 & 0 \\
\hline Adoquín & 6,7 & 3,7 & 11,1 \\
\hline $\begin{array}{l}\text { Nódulos (Tamaño } \\
\text { entre 7-30 mm) }\end{array}$ & 6,7 & 3,7 & 11,1 \\
\hline Lineal & 6,7 & 7,4 & 5,5 \\
\hline
\end{tabular}

Fuente: autores.
Las dos distribuciones más frecuentes de los hallazgos semiológicos en la tomografía se encuentran en las regiones inferiores, teniendo un compromiso difuso, de igual manera al realizar el análisis por género (Ver Figura 7). Sin embargo, aquí no se observa predominio difuso en la población femenina como se describió en los estudios radiográficos.

Los hallazgos en tomografía tienen una localización predominantemente bilateral $(60 \%$ de los casos analizados) (Ver Figura 8).

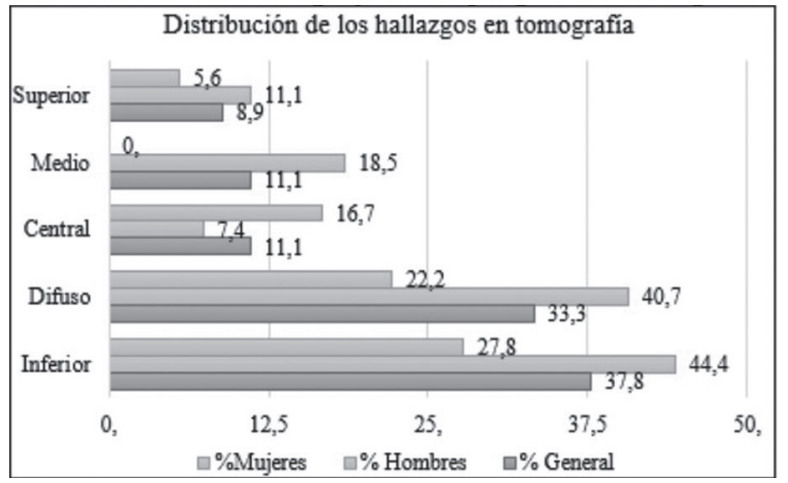

Figura 7. Distribución de los hallazgos y análisis por género en tomografía

Fuente: autores.

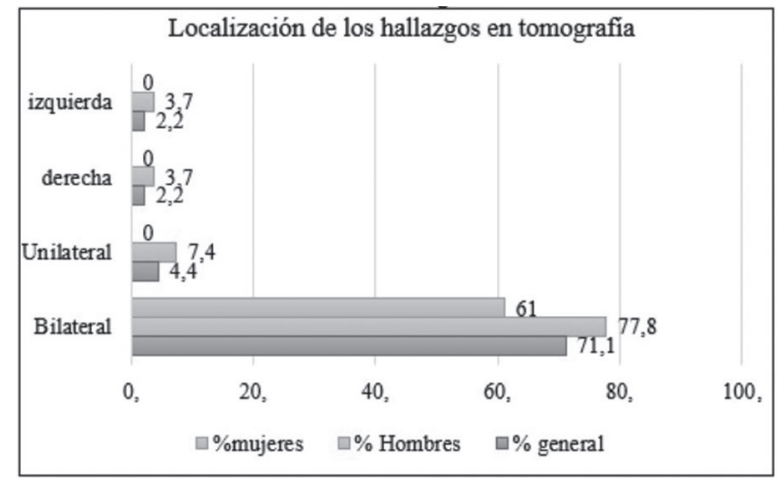

Figura 8. Análisis de la localización en la tomografía.

Fuente: autores.

\section{Discusión}

Esta es la primera revisión que intenta recopilar la mejor evidencia posible para determinar los hallazgos más frecuentes, las localizaciones y distribución de las anormalidades en radiografía y tomografía como estándares del estudio imagenológico, la mayoría de las mismas en un contexto agudo (bien sea por presentación inicial o reagudización en casos crónicos). 
Teniendo en cuenta los estudios radiográficos se puede decir que el hallazgo más frecuente de la hemosiderosis pulmonar idiopática en nuestro estudio es la consolidación y las opacidades reticulonodulares. Otro aspecto importante es que lo menos reportado es la opacidad en vidrio deslustrado. Las anormalidades radiográficas se distribuyen predominantemente en el tercio inferior y de manera difusa, siendo más frecuente esta última en la población femenina y finalmente la afección bilateral es la más encontrada independiente del género. Lo anterior en concordancia con las observaciones descritas por Khorashadi et $\mathrm{al}^{2}$ en donde dice que las manifestaciones que afectan al individuo en la fase aguda, las cuales típicamente en las radiografías de tórax se caracterizan por opacidades y consolidación de predominio hiliar, perihiliar y de los lóbulos inferiores, usualmente los ápices se respetan. Por la evolución natural de la enfermedad en el tiempo, las consolidaciones inicialmente encontradas en los pacientes tienden a desaparecer en los primeros 3 días de la presentación y las opacidades reticulares llegan a ser evidentes². Hasta un $5 \%$ de las radiografías son reportadas como normales en nuestro análisis.

En cuanto a la tomografía, el hallazgo más frecuente es la presencia de vidrio deslustrado con más de $50 \%$ de los casos y consolidaciones en más de $40 \%$. Las anormalidades se distribuyen en las porciones basales (inferiores) y de forma difusa, en este mismo orden, tanto para hombres como mujeres, finalmente la afección es bilateral en más del $60 \%$ de los casos. Observaciones concordantes con las de Ioachimanescu et al. y Khorashadi ${ }^{2,3}$.

En otros hallazgos ninguna tomografía se reportó como normal, se encontraron quistes hasta en un $8,9 \%$ y panalización hasta en $6,7 \%$, probablemente debido a la presencia de la enfermedad desde la infancia, la cual lleva a cambios cicatriciales en pacientes con episodios repetidos de hemorragia alveolar, lo cual puede desencadenar fibrosis pulmonar que en radiografía se manifiestan como opacidades reticulares basales y subpleurales².

Se debe tener en cuenta que ninguno de los porcentajes expresados en los hallazgos de imagen va a tener como valor final de la sumatoria $100 \%$ porque las categorías no son mutuamente excluyentes y muchas manifestaciones pueden encontrarse en un mismo paciente.
Una limitación importante de las publicaciones analizadas es que no hay metodología hasta el momento que pueda evaluar la calidad de los artículos recopilados de manera objetiva, como sí lo hacen para otro tipo de estudios como ensayos clínicos o estudios de cohorte; sin embargo, y dada la frecuencia de la enfermedad en la población, la situación se limita al reporte de casos, revisiones y series de casos en la literatura, se considera entonces que se está evaluando la mejor evidencia posible. En algunas descripciones no se hacía especificidad de los hallazgos de acuerdo con las categorías mencionadas, esto como se dijo, por la evolución natural de la literatura y el conocimiento médico. Asimismo, se mencionaban "opacidades" o "infiltrados" sin determinar la localización anatómica, lo que hizo necesario la interpretación de los hallazgos de acuerdo con las imágenes presentadas en el artículo por parte de los autores, lo que podría configurar un sesgo.

Se intentó analizar la raza y la profesión, para después establecer si había alguna relación con los hallazgos, sin embargo, solo 3 estudios: Yanagihara T et al. ${ }^{7}$, Mayes et al. ${ }^{26}$ y Chen et al. ${ }^{28}$ la incluyeron. Se consideró inicialmente hacer una comparación sobre la técnica empleada en el estudio tomográfico de tórax convencional vs alta resolución, sin embargo, no había especificidad de la técnica en los estudios de una manera puntual, por lo que se decidió dejar atrás esta aclaración. Otras variables como síntomas clínicos, tratamiento y respuesta también fueron omitidos, debido a la existencia en la literatura de un reporte concentrado en dichos aspectos ${ }^{48}$.

\section{Conclusiones}

La hemosiderosis pulmonar idiopática en radiografía presenta un compromiso por consolidación o reticulonodular en mayoría de los casos. Las afecciones principalmente se localizan de manera bilateral distribuyendose en las regiones inferiores o de manera difusa, ésta última característica predomina en las mujeres. En tomografía el hallazgo más frecuentemente reportado es la opacidad en vidrio deslustrado y las consolidaciones que afectan a los segmentos inferiores y se distribuyen de manera difusa en ambos parénquimas pulmonares en más del $60 \%$ de los casos. Curiosamente, en las mujeres solo se reportaron casos bilaterales en la tomografía. 


\section{Referencias bibliográficas}

1. Agarwal R, Aggarwal AN, Gupta D. Lane-Hamilton syndrome: simultaneous occurrence of coeliac disease and idiopathic pulmonary haemosiderosis. Intern Med J. 2007;37(1):65-7.

2. Khorashadi L, Wu C, Betancourt S, Carter B. Idiopathic pulmonary haemosiderosis: spectrum of thoracic imaging findings in the adult patient. Clinical Radiology. 2015;70(5):459-65.

3. Ioachimescu OC, Sieber S, Kotch A. Idiopathic pulmonary haemosiderosis revisited. Eur Respir J. 2004;24(1):162-70.

4. Fleischner FG, Berenberg AL. Idiopathic pulmonary hemosiderosis. Radiology. 1954;62(4):522-6.

5. Gencer M, Ceylan E, Bitiren M, Koc A. Two sisters with idiopathic pulmonary hemosiderosis. Can Respir J. 2007;14(8):490-3.

6. Berger N, Nichols J, Datta D. Idiopathic pulmonary haemosiderosis with celiac disease (Lane-Hamilton syndrome) in an adult - a case report. Clin Respir J. 2015;10(5):661-5.

7. Yanagihara T, Yamamoto Y, Hamada N, Suzuki K, Ogata-Suetsugu S, Harada E, et al. Recurrent idiopathic pulmonary hemosiderosis after long-term remission presented with Sjogren's syndrome: Idiopathic no more? Respir Med Case Rep. 2018;25: 68-72.

8. Patiño S, Moncada DC, Ramírez JD. Hemosiderosis pulmonar idiopática en paciente adulto. Acta Med Colomb. 2017;42(1):64-7.

9. Silva P, Ferreira PG. Idiopathic pulmonary hemosiderosis: Hemorrhagic flare after 6 years of remission. Rev Port Pneumol. 2017;23(6):368-73.

10. Popp A, Jurcut C, Vasile D, Sotcan M,Laurila K, Jinga M. Severe Alveolar Hemorrhage - What's in it for the Gastroenterologist? JGLD. 2016;25(4):555-8.

11. Barrera AM, Vargas L. Idiopathic pulmonary hemosiderosis with dendriform pulmonary dossification. Biomédica.2016;36(4):504.

12. Cambruzzi E, Pêgas KL, Vedana T. Idiopathic pulmonary hemorrhage: morphology and differential diagnosis. Case report J Bras Patol Med Lab. 2013;49(3):216-21.

13. Patrucco F, Sarcoli M, Boldorini R, Balbo PE. a young man with anemia and recurrent tachyarrhythmic episodes. Respiration. 2013;86(2):149-154.

14. Törö K, Herjavecz I, Vereckei E, Kovács M. Fatal idiopathic pulmonary haemosiderosis in association with pregnancy medico-legal evaluation. J Forensic Leg Med. 2012;19(2):101-4.

15. Santos JWAD, Neto ABDM, Marchiori RC, Michel GT, Fagundes AL, Tagliari LGM, et al. Hemossiderose pulmonar associada à doença celíaca: melhora após dieta livre de glúten. J Bras Pneumol. 2012;38(3):412-4.

16. Miwa S, Imokawa S, Kato M, Ide K, Uchiyama H, Yokomura $\mathrm{K}$, et al. Prognosis in adult patients with idiopathic pulmonary hemosiderosis. Intern Med. 2011;50(17):1803-8.

17. Lang L,Wang X,Hou W. Successful treatment of adult idiopathic pulmonary hemosiderosis with integrated traditional Chinese and Western medicine: a report of one case. J Integr Med. 2011;9(5):5312.

18. Persson HL, Vainikka LK, Eriksson HB, Wennerström U. LaneHamilton syndrome: ferritin protects lung macrophages against iron and oxidation. Chest. 2011;139(2):361-7.

19. Oviedo Ramírez MI, Hop K, Carrera E, Soriano Rosas J. Hemosiderosis pulmonar idiopática en un adulto joven. Hallazgos de autopsia. Arch Bronconeumol. 2010;46(10):565-7.

20. Krumsiek A, Poggemann V, Wertzel H, Achenbach HJ. Rezidivierende Hämoptysen bei einer 29-jährigen Patientin. Der Internist. 2010;51: 1561-6.

21. Robles M, Torres J, Rojas A. Tratamiento anestésico para cesárea electiva en una paciente con hemosiderosis pulmonar idiopática. Rev Esp Anestesiol Reanim. 2009;56(9): 577-8.

22. Mu XD, Su L, Nie LG, Na J, Wang RG, Li HC. Idiopathic pulmonary hemosiderosis in adults: report of two cases and literature review. Beijing Da Xue Xue Bao Yi Xue Ban. 2008;40(6): 595-9.

23. Harte S, Mcnicholas WT, Donnelly SC, Dodd JD. Honeycomb cysts in idiopathic pulmonary haemosiderosis: high-resolution CT appearances in two adults. B J Radiol. 2008;81(972): e295-8.

24. Foglia LM, Deering SH, Foglia LM, Deering SH. Post-partum exacerbation of idiopathic pulmonary hemosiderosis. J Matern Fetal Neonatal Med. 2008;21(12): 895-7.
25. Souza BV de, Schwingel FL, D' Aquino LC. Hemosiderose pulmonar idiopática: exacerbação associada à gestação. Rev Soc Bras Clín Méd 2008;6(4): 160-2.

26. Mayes DH, Guerrero ML. A few good men: a marine with hemoptysis and diarrhea. idiopathic pulmonary hemosiderosis and celiac sprue. Chest. 2008;134(3):644-7.

27. Allen TK, George RB, Peterson-Layne C, Habib AS. Management of a parturient with an acute exacerbation of idiopathic pulmonary haemosiderosis and posterior spinal instrumentation. Br J Anaesth. 2008;100(2): 235-9.

28. Chen C-H, Yang H-B, Chiang S-R, Wang PC. Idiopathic pulmonary hemosiderosis: favorable response to corticosteroids. J Chin Med Asoc. 2008;71(8) 421-4.

29. Inayama $\mathrm{M}$, Hino $\mathrm{H}$, Takezaki $\mathrm{A}$, Otsuka $\mathrm{S}$, Machida $\mathrm{H}$, Hatakeyam N, et al. A case of adult onset idiopathic pulmonary hemosiderosis markedly improved by steroid therapy. Nihon Kokyuki Gakkai Zasshi. 2007; 45(12):971-6.

30. Deniz O, Ongürü O, Ors F, Gümüş S, Tozkoparan E, Bilgiç H, et al. Idiopathic pulmonary hemosiderosis in an adult patient responded well to corticosteroid therapy. Tuberk Toraks. 2007; 55(1):77-82.

31. Vinodh BN, Sharma SK, Mukhopadhyay S, Ray R. Idiopathic pulmonary haemosiderosis: two case reports. Indian J Chest Dis Allied Sci. 2006;48(1): 75-7.

32. Ioachimescu OC, Jennings C. Intercostal lung cyst hernia in idiopathic pulmonary hemosiderosis (cyst necessitans). Mayo Clinic Proc. 2006;81(5): 692.

33. Malhotra P, Aggarwal R, Aggarwal AN, Jindal SK, Awasthi A, Radotra BD. Coeliac disease as a cause of unusually severe anaemia in a young man with idiopathic pulmonary haemosiderosis. Respir Med. 2005;99(4):451-3.

34. Soto RG, Soares MM. Idiopathic pulmonary hemosiderosis in pregnancy: anesthetic implications. J Clin Anesth. 2005;17(6): $482-4$.

35. Maalej S, Drira I, Fennira H, Ben Mefteh R, Bourguiba M, Zidi A, et al. Hémosidérose pulmonaire idiopathique de l'adulte. Rev Pneumol Clin. 2005; 61(2):109-11.

36. Turay UY, Ergün P, Erdoğan Y, Biber C, Demirağ F, Keyf IA. Idiopathic pulmonary haemosiderosis. Tuberk Toraks 2004; $52(4): 382-5$.

37. Helman DL, Sullivan A, Kariya ST, Deering SH, Hueppchen NA, Shorr AF. Management of idiopathic pulmonary haemosiderosis in pregnancy: Report of two cases. Respirology. 2003; 8(3):398400 .

38. Calabrese F, Giacometti C, Rea F, Loy M, Sartori F, Di Vittorio $\mathrm{G}$, et al. Recurrence of idiopathic pulmonary hemosiderosis in a young adult patient after bilateral single-lung transplantation. Transplantation. 2002;74(11):1643-5.

39. Tedeschi A, Lorini M, Giannini S, Ciceri L, Suli C, Airaghi L. Serum histamine-releasing activity in a patient with idiopathic pulmonary haemosiderosis. Allergol Immunopathol (Madr). 2001;29(6):281-3.

40. Kishimoto N, Kondou H. A case of idiopathic pulmonary hemosiderosis of adult onset. Nihon Kokyuki Gakkai Zasshi. 2000;38(8):589-93.

41. Ruchin P, Robinson J, Segasothy M, Morey F. Melioidosis in a patient with idiopathic pulmonary haemosiderosis resident in Central Australia. Aust N Z J Med. 2000;30(3):395-6.

42. Godoy I, Leite RM, Yoo HH, Defaveri J, Catâneo AJ, Queluz TH. Idiopathic pulmonary hemosiderosis with cystic lesions: a rare presentation. Am J Med Sci. 2000;319(6):411-3.

43. Bavry AA, Knoper S, Alpert JS. Segmental Wall Motion Abnormalities in an Individual with Idiopathic Pulmonary Hemosiderosis. Cardiology. 2000;93(3):201-4.

44. Ramírez T, Prados C, Gómez de Terreros Caro J, Villamor J, Álvarez-Sala R. Hemosiderosis pulmonar idiopática en paciente de edad avanzada. Arch Bronconeumol. 1999;35(10):507-9.

45. Hansell DM, Bankier AA, MacMahon H, McLoud TC, Müller NL, Remy J. Fleischner society: glossary of terms for thoracic imaging. 2008; 246(3):697-722.

46. Webb WR, Higgins CB. Thoracic Imaging: Pulmonary and Cardiovascular Radiology. 3rd ed. Wolters Kluwer; 2016.

47. Khorashadi L, Wu CC, Betancourt SL, Carter BW. Idiopathic 
Muñoz Durán JA, Muñoz Durán AF

pulmonary haemosiderosis: spectrum of thoracic imaging findings in the adult patient. Clin Radiol. 2015; 70(5):459-65.

48. Chen X-Y, Sun J-M, Huang X-J. Idiopathic pulmonary
MÉD.UIS. 2020;33(2):55-64

hemosiderosis in adults: review of cases reported in the latest 15 years: Idiopathic pulmonary hemosiderosis in adults. Clin Respir J. 2017;11(6):677-81 